\title{
Determinación de la eficiencia de un producto fijador para la protección de tintas ferrogálicas
}

\author{
ARIEL VIZCAÍNO', TROSKY YÁNEZ2 ${ }^{*}$, PABLO BONILLA ${ }^{3}$ \\ 1. Universidad Central del Ecuador, Acervo Histórico, Laboratorio Análisis Químico. \\ 2. Universidad Central del Ecuador, Facultad de Ciencias Químicas, Laboratorio de Química Orgánica. \\ 3. Universidad Central del Ecuador, Facultad de Ciencias Químicas, Laboratorio de Nanotecnología. \\ Correspondencia: tgyanez@uce.edu.ec,ariwy90@hotmail.com
}

Recibido: 8 septiembre 2017. Aceptado: 20 noviembre 2017

\section{Resumen}

El objetivo de esta publicación es divulgar los resultados de la formulación y evaluación de un fijador alternativo de tintas ferrogálicas desarrollado en el Área Histórica de la Universidad Central del Ecuador, el mismo que es utilizado para proteger el contenido de documentos patrimoniales. Se usó como componentes al poliestireno, di(2-etil-hexil) ftalato (DOP) y aceite de naranja, evitando así el empleo de solventes aromáticos. Para su evaluación se cuantificó el contenido de hierro mediante absorción atómica presente en el agua residual del tratamiento que regula el pH de un documento, obteniéndose como resultado que no se determinaron contenidos de hierro en los escritos en los que se aplicó el fijador. También se analizó el efecto del fijador en los documentos considerando el antes y despúes del uso del fijador, con la utilización de una encuesta evaluada por 20 expertos en conservación, en la que se examinaron parámetros como el color e intensidad de la escritura, presencia de brillo y de sustancias extrañas, concluyendo que el $82 \%$ de los encuestados no observaron cambios en el color e intensidad, el 83\% estableció que no existe brillo al aplicarse el fijador y finalmente el $77 \%$ señaló que existe reducción en la presencia de manchas.

Palabras clave: Tintas ferrogálicas, fijador de finta, poliestireno, dioctil-ftalato, aceite de naranja.

\section{Determination of the efficiency of a fixing product for the protection of irongall inks}

\section{Abstract}

The objective of this publication is to disseminate the results of the formulation and evaluation of an alternative fixer of iron-gall inks developed in the Historical Area of the Central University of Ecuador, which is used to protect the content of patrimonial documents. Polystyrene, di (2-ethylhexyl) phthalate (DOP) and orange oil were used as components, thereby avoiding the use of aromatic solvents. To evaluate it, the iron content was quantified by atomic absorption, present in the residual water of the treatment that regulates the $\mathrm{pH}$ of a document, as a result, iron contents were not found in the documents in which the fixer was applied. The effect of the fixer on the documents was also analyzed considering a before and after use of the fixer, a survey administered to 20 conservation experts, in which parameters such as the color and intensity of the documents, presence of brightness and foreign substances, concluded that $82 \%$ of the respondents did not observe changes in color and intensity, $83 \%$ affirmed there is no shine when applying the fixer, and finally $77 \%$ determined a reduction in the presence of spots.

Keywords: Inks, ink fixer, polystyrene, dioctyl phthalate, orange oil. 


\section{Introducción}

Uno de los aspectos más preocupantes y que afectan específicamente a la información contenida en los documentos, es la presencia de humedad que genera la aparición de microorganismos, así como el deterioro de las tintas que puede producir o acelerar efectos corrosivos en el soporte por acidez de la tinta, contribuyendo a la hidrólisis y/o degradación oxidativa de la celulosa.

Durante el proceso de conservación y/o restauración de libros, se llevan a cabo diversos tratamientos que se deben hacer al documento para evitar, mitigar o eliminar las afectaciones señaladas, pudiendo ser estos procesos acuosos y no acuosos [1]. Cuando se debe someter al documento a un tratamiento acuoso, como lavados con soluciones buffer que regulan el $\mathrm{pH}$ en el papel, si hay presencia de tinta ferrogáli$\mathrm{ca}$, es necesario la protección de la misma debido al desprendimiento del hierro por su solubilidad en compuestos polares.

Actualmente para la protección de la tinta ferrogálica [2] [3] se utiliza un tipo de resina acrílica conocida comercialmente como Paraloid B-72 [4], el cual da la protección no solo a tintas acuosas, sino también a objetos de cerámica que entran a procesos de conservación [5]. La gran dificultad que presenta este fijador es que la resina acrílica es soluble en compuestos orgánicos levemente polares, siendo así necesario el uso de compuestos muy tóxicos como el xileno, tolueno $y$ thinner [6], al igual que otros fijadores usados en menor grado como el acetato de celulosa y el acetato de polivinilo que también son solubles en solventes tradicionales como la acetona o el xileno.

Por este motivo en esta investigación, se buscó formular y evaluar un producto fijador alternativo que contenga sustancias menos tóxicas, permitiendo una adecuada protección de las tintas ferrogálicas durante una etapa acuosa de tratamiento como la regulación de $\mathrm{pH}$, y tomando en cuenta la estética y la textura que puede dejar sobre el documento al final.

Se ha elegido el poliestireno [10] como un polímero de bajo costo que puede resultar estable, ligero y traslúcido para el efecto, junto con el di(2-etil-hexil) ftalato o llamado comercialmente dioctil-ftalato (DOP) [11] que es usado como plastificante, el cual permite mejorar la flexibilidad del polímero, y el aceite de naranja [7] [8] [9] como solvente alternativo de baja toxicidad para la mezcla de las sustancias.

\section{Materiales y Métodos}

Para la determinación de hierro que puede aparecer desprendido en el líquido usado en los procesos de lavado de un documento, se utilizaron treinta hojas de papel de pasta química o "papel bond" tomadas como la totalidad de la muestra, y para el levantamiento de la encuesta se aplicó el producto fijador en un documento proporcionado por el Área Histórica, sobre el cual veinte personas expertas en conservación pertenecientes al Área Histórica del Archivo General de la Universidad Central, Casa de la Cultura Ecuatoriana y Ministerio de Cultura, valoraron la estética del documento antes y después de su aplicación.

\section{Etapa I:}

Se realizaron diversas combinaciones en la preparación del producto fijador variando la concentración del poliestireno tomado como capa protectora, al DOP como plastificante y al aceite de naranja como solvente, hasta encontrar la concentración adecuada a través de un diagrama de fases, evaluando que fije la tinta con el soporte, que no deje manchas en las hojas al aplicarse y considerando que la textura tampoco sea alterada.

\section{Etapa II:}

Se preparó tinta ferrogálica de composición definida usando los materiales básicos que son conocidos bibliográficamente, como el extracto de taninos, sulfato ferroso, goma arábica y agua [12]. Se tomó un mililitro de tinta ferrogálica preparada y se aplicó en hojas de "papel bond" con la ayuda de un pincel número 1 , se repitió este proceso en un total de treinta hojas. Una vez seca la tinta se procedió consolidar con el fijador alternativo en un total de quince hojas, para continuar con la simulación del proceso de lavado tradicional realizado en los tratamientos de conservación, considerando que el pH del agua este cercano a 7, su temperatura sea la de laboratorio y el tiempo de sumergido de 5 minutos. Concluido ese tiempo se tomó una muestra del agua al final de este proceso y se procedió a determinar el hierro presente en el líquido por el método de absorción atómica, el cual es un método de análisis que esta validado y acreditado en el laboratorio de la Facultad de Ciencias Químicas de la Universidad Central del Ecuador.

\section{Etapa III:}

Se obtuvo un documento del archivo proporcionado por el Área Histórica de la Universidad Central del Ecuador, el cual fue fotografiado mediante un equi- 
po de barrido digital marca Scan Diva en un área determinada antes de aplicar el fijador y se volvió a realizar el barrido digital reproduciendo las mismas condiciones (distancia, ángulo, área) de la fotografía anterior una vez que se aplicó el producto fijador. Posteriormente se presentó una encuesta con tres pares de fotografías para que sea evaluado en función del cambio de color e intensidad de la grafía, pre- sencia de brillo en la grafía, y la presencia de sustancias extrañas (desprendimiento de hierro alrededor de la grafía incluido manchas) antes y después de aplicar el fijador (Fig. 1). Estos parámetros de evaluación fueron sugeridos por los propios expertos en conservación como los más relevantes al momento de analizar la alteración de un documento por la acción de un fijador.

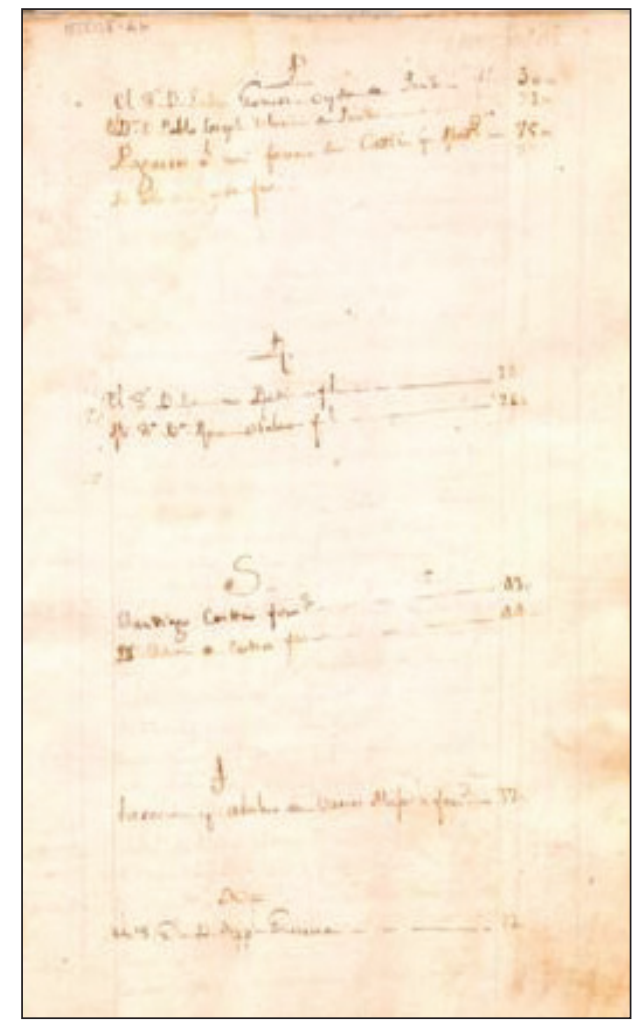

Fotografía "antes" (Sin fijador)

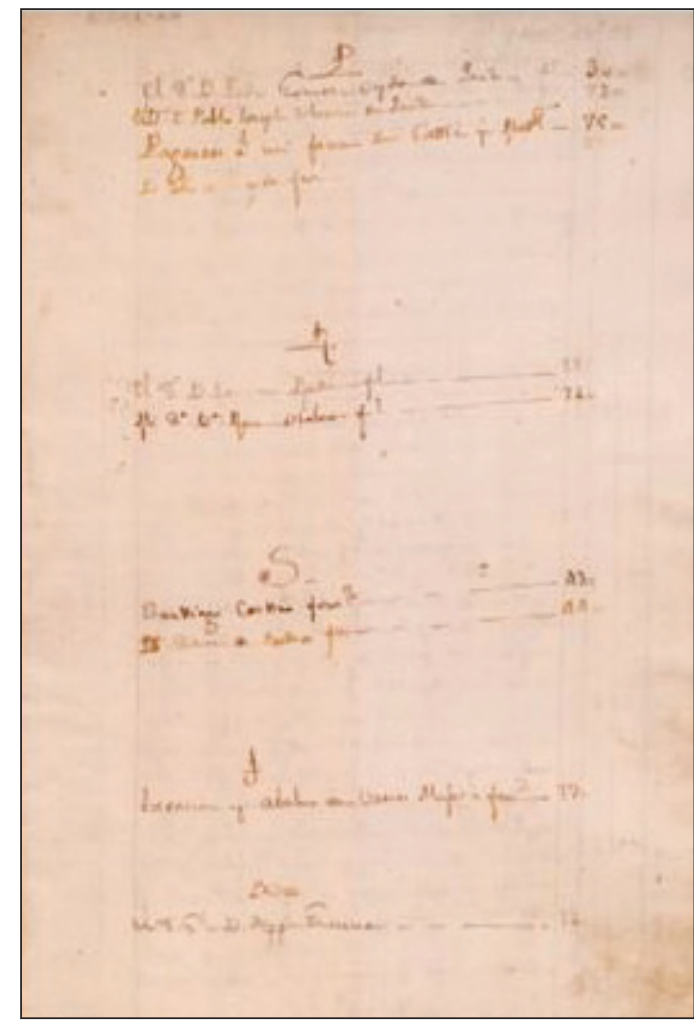

Fotografía "después" (Con fijador)

Figura 1. Ejemplar de un para de fotografías evaluando mediante la encuesta a expertos.

En esta etapa de valoración cualitativa, la encuesta fue entregada a 20 personas involucradas en el proceso conservación de documentos y de pinturas, para que asigne un parámetro de cualidad del documento respecto al color y presencia de brillo en la grafia y presencia de cuerpos extraños incluido manchas.

Cada parámetro tiene un valor arbitrario que va de 1 (No existe diferencia) hasta 5 (Completamente diferentes), Tabla 1.

Cada uno de estos valores arbitrarios se multiplica por el número de encuestados (20 expertos) para obtener así el valor total para cada parámetro (rango de valor).
Tabla 1. Relación de los parámetros de la encuesta con un rango de valores.

\begin{tabular}{cccc}
\hline Parámetro & $\begin{array}{c}\text { Valor } \\
\text { Arbitrario }\end{array}$ & $\begin{array}{c}\text { Numero } \\
\text { de } \\
\text { encuestados }\end{array}$ & $\begin{array}{c}\text { Rango } \\
\text { de } \\
\text { valor }\end{array}$ \\
\hline $\begin{array}{c}\text { No existe } \\
\text { diferencia } \\
\text { Poca } \\
\text { diferencia }\end{array}$ & 1 & & $20-39$ \\
Mediana \\
diferencia \\
Alta \\
diferencia
\end{tabular}




\section{Resultados y Discusiones}

\section{Etapa I:}

Para la determinación de cada componente del fijador alternativo se tomó en cuenta que estos no causen manchas al momento de aplicarlo en el documento una vez que este seco, y que además no cambie la coloración de la tinta, siendo la composición ideal del fijador de $1 \%$ poliestireno y $0.6 \%$ de DOP.

\section{Etapa II:}

Durante el proceso de lavado se pudo apreciar que los documentos en los que se aplicó el fijador, la tinta no entró en contacto directo con el agua por alrededor de $4 \mathrm{~min}$, esto es debido a que el fijador tiene una acción impermeabilizante. También se observó que al momento de retirar el documento del agua no quedan residuos de la tinta $(\mathrm{Fe})$ en el fondo del recipiente, esto se cumple para aquellos documentos que fueron tratados con el fijador alternativos figura 2- $\mathrm{A}$, al contrario de los documentos en los que no se utilizó el fijador figura 2-B donde se aprecia fragmentos negros en la parte inferior de la fotografía. Esto ocurre debido a que los documentos sin tratamiento tienen un contacto directo con el agua lo cual provoca que la tinta sea fácilmente desprendida.
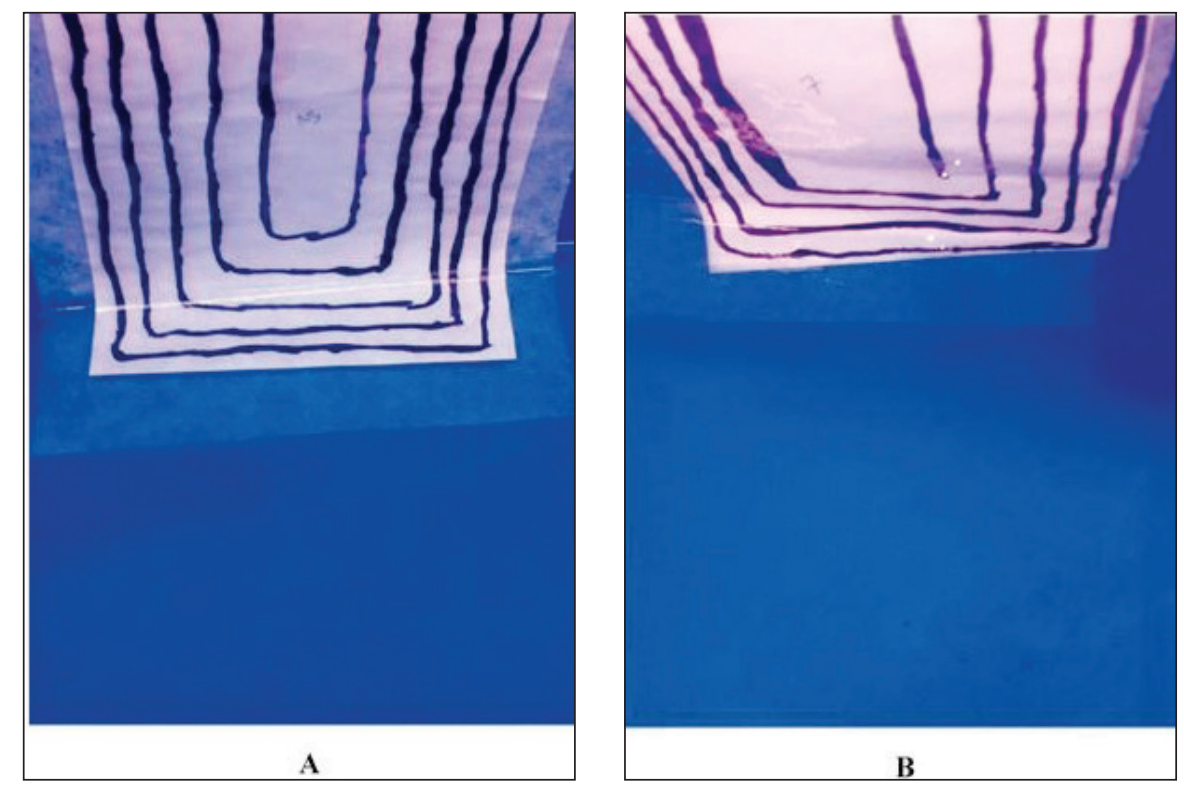

Figura 2. Documento una vez sometido al lavado.

Cabe señalar que una vez que el documento sometido al proceso de lavado para el control del pH se seca, también se aprecia el desprendimiento de tinta ferrogálica en el papel por la aparición de una cierta escarcha metálica esparcida en su superficie, este hecho fue valorado en la encuesta al preguntar si el experto ve la presencia de cuerpos extraños en el documento.

Adicionalmente para establecer cuantitativamente el desprendimiento de hierro en el agua de lavado se recolectaron 30 muestras que fueron analizadas por el método de absorción atómica, método instrumental validado que posee un límite de cuantificación de 0.07 ppm y un coeficiente de correlación de 0.998 . En este análisis los resultados promedio obtenidos para los documentos sin fijador fueron de un valor de
$0.27 \mathrm{ppm}$ de hierro con una desviación estándar de 0.06 ppm, y por debajo del límite de cuantificación del equipo cuando se utilizó el fijador alternativo.

\section{Etapa III:}

\section{- Color e intensidad de la grafía}

La diferencia de color en la grafía obtuvo en puntaje final promedio para las fotografías presentadas de 50 puntos, al ubicar este valor en la tabla 1, se establece el parámetro para esta característica como "Poca diferencia", es decir existe poca diferencia entre el antes y después del tratamiento de conservación con la utilización de fijador, hecho evaluado usando fotografías reales de un libro. 
En la figura 3 se puede observar los resultados de la encuesta a expertos para este parámetro, realizado en los tres pares de fotografías. En este análisis se observa que los encuestados asignaron valores de $85 \% ; 65 \%$ y $95 \%$ (promedio $82 \%$ ) a aquellas imágenes que corres- ponden al documento tratado con fijador y que presentan una mayor tonalidad en la grafía en comparación a los documentos que no recibieron este tratamiento. Los pares de fotografías tratadas con fijador corresponden a las $B, A$ y $B$ de la figura 3 respectivamente.

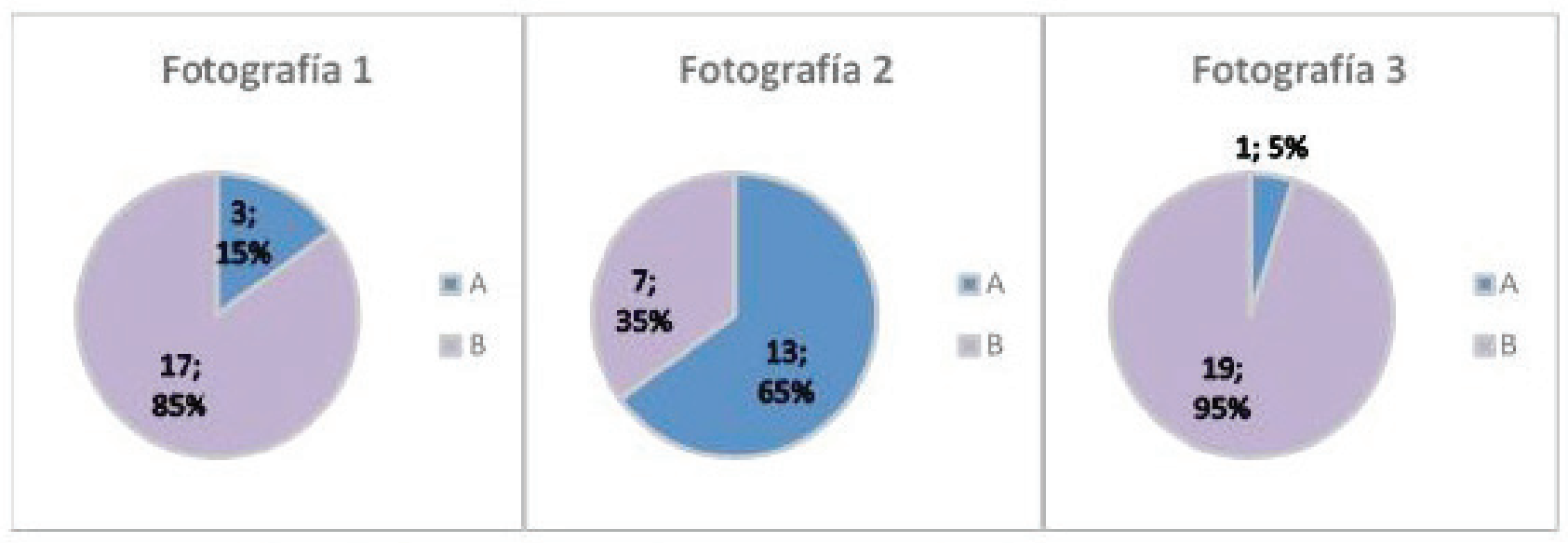

Figura 3. Fotografía con mayor intensidad de color en caligrafía.

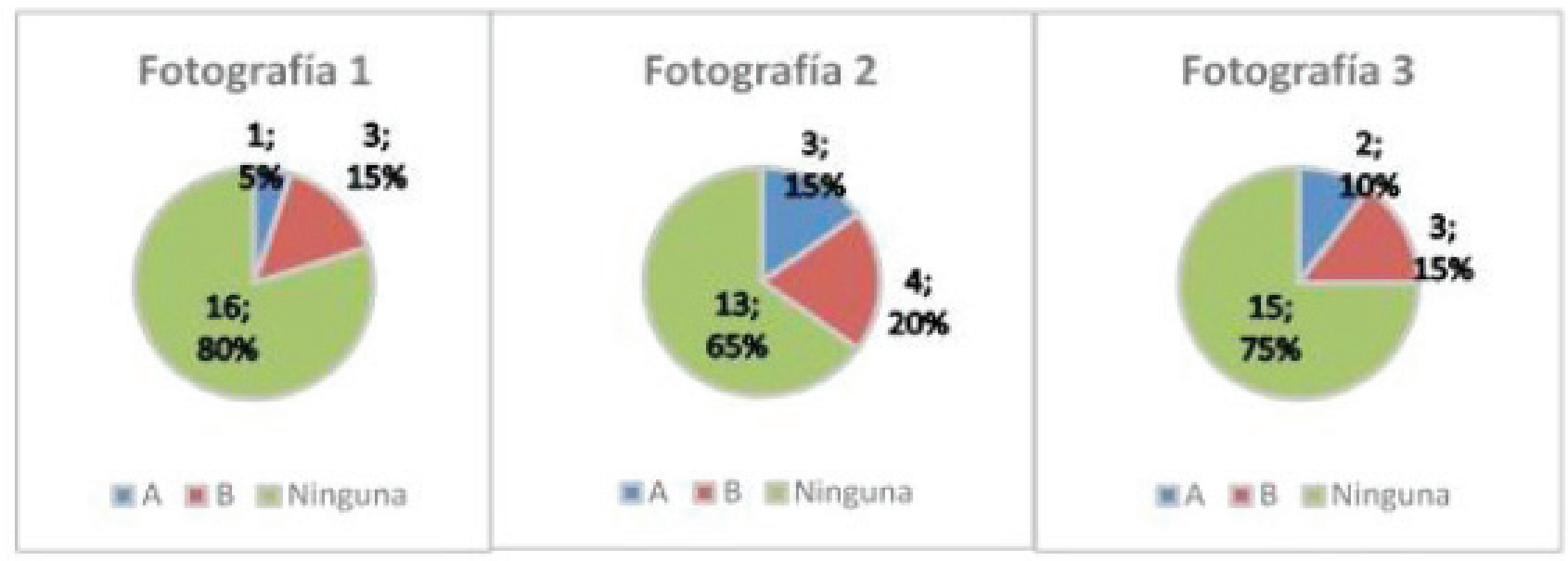

Figura 4. Evaluación del brillo en las fotografías antes y después del fijador.

- $\quad$ Presencia de brillo en la grafía

La presencia de brillo en la grafía obtuvo en puntaje final promedio para las fotografías presentadas de 30 puntos, al comparar este valor con la tabla 1, se establece que el parámetro para esta característica es de "No existe diferencia", es decir no hay diferencia entre el antes y después del tratamiento de conservación ("ninguna diferencia": 80\%, 65\%,75\%) figura 4 .

- Presencia de sustancias extrañas

Finalmente la evaluación sobre la presencia de sustancias extrañas obtuvo en puntaje final promedio para las fotografías presentadas de 42 puntos, al ubicar este valor en la tabla 1, se establece el parámetro para esta característica como "Poca diferencia", es decir existe poca diferencia entre el antes y después del tratamiento de conservación con la utilización del fijador.

En la figura 5 se ilustra el resultado de los expertos respecto a la presencia de manchas o suciedad. En este análisis se observa que los encuestados asignaron valores de 60\%, $85 \%$ y $85 \%$ (promedio $77 \%$ ) para las fotografías $A, B$ y $A$ respectivamente, que corresponden a 
las instantáneas de los documentos que fueron tomados antes de realizar la fijación de la tinta.

Esta diferencia respecto a la apreciación de las fotografias posteriores a la aplicación del fijador según los encuestados permite concluir que al someter el documento al proceso de lavado las manchas son eliminadas.

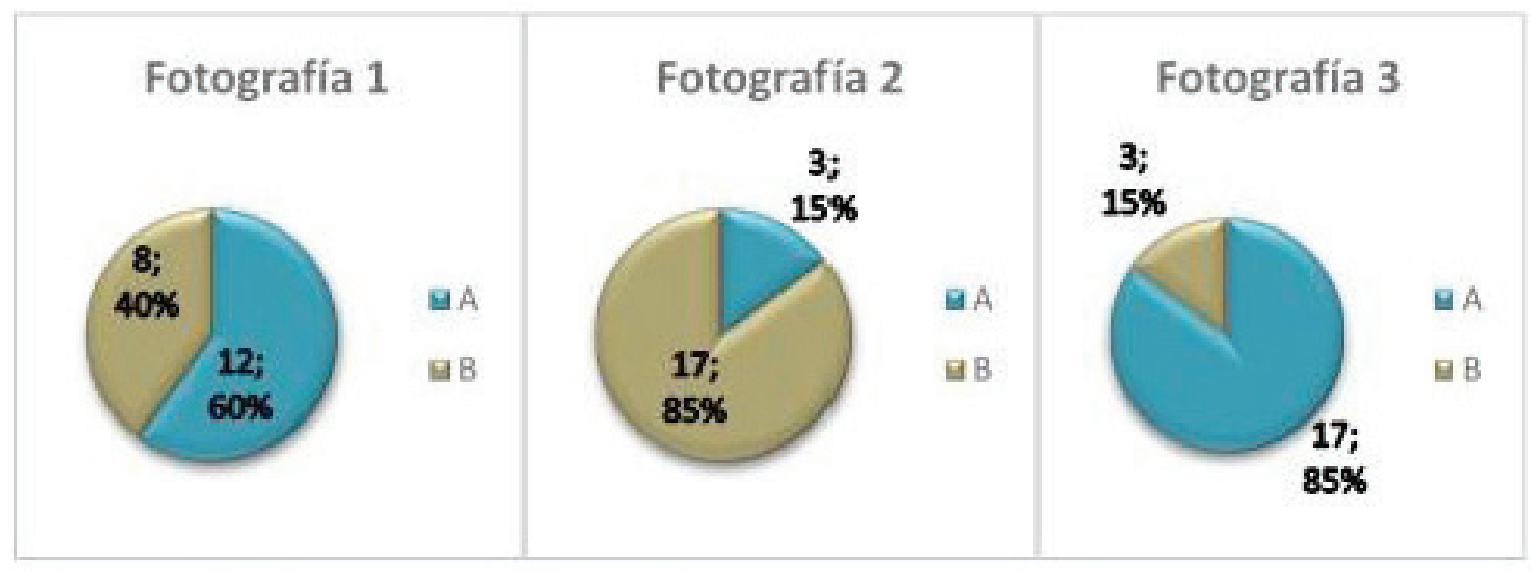

Figura 5. Porcentajes de sustancias extrañas en los documentos.

\section{Conclusiones}

- La formulación ideal del producto fijador de tintas ferrogálicas se elaboró con $1 \%$ de poliestireno, $0.6 \%$ de di(2-etil-hexil) ftalato (DOP) y aceite de naranja como solvente, lo cual modifica sustancialmente la composición de los productos tradicionalmente usados para este efecto sobre todo el empleo de solventes arómáticos.

- La evaluación de la eficiencia del producto por el método de absorción atómica fue adecuado y confiable ya que permitió determinar la cantidad de hierro desprendido en el líquido de lavado, cuando los documentos tratados están con y sin fijador. En esta valoración se determinó un valor promedio de 0.27 ppm para las muestras líquidas provenientes de documentos sin fijador e indeterminado o sea debajo del límite de cuantificación del equipo, para aquellas muestras líquidas en las cuales si se aplicó el producto de sujeción.

- Los resultados obtenidos en la encuesta de evaluación del cambio de color e intensidad en la grafía, fue que existe poca diferencia entre los pares de fotografías tomadas antes y después de la aplicación del fijador respecto al color de la escritura, y que se observa mayor tonalidad en aquellas tomadas después del proceso de fijación con un valor de aceptación promedio del $82 \%$.

Los resultados alcanzados en el estudio de opinión respecto a la presencia de brillo en la escritura, fue que no existe diferencia entre los pares de fotografías presentadas antes y después del tratamiento de conservación. La valoración obtenida para este parámetro fue del $83 \%$ que establece que ninguna fotografía presenta brillo.

- Los resultados obtenidos por la encuesta en la evaluación de presencia de sustancias extrañas y/o manchas, fue que existe poca diferencia en las fotografías tomadas antes y después de aplicar el fijador en un proceso de conservación acuoso, por consiguiente, las instantáneas con mayor presencia de sustancias extrañas $y / 0$ manchas fueron las tomadas antes del proceso, con una apreciación promedio del $77 \%$ para las fotografías con 
mayor presencia de sustancias extrañas sobre el soporte, lo que sugiere que las fotografías después de la aplicación del fijador no presentaron escarcha y las manchas se reducen al pasar por el tratamiento de lavado.
De conformidad a los parámetros valorados, se concluye que el producto elaborado no afecta las características visuales del documento de acuerdo con el criterio de los expertos en conservación encuestados.

\section{Referencias}

[1] C. C. y. V. Viñas, «Las Tintas, Elementos graficos del documento,» de La preservación y restauración de documentos y libros en papel: un estudio del RAMP con directrices., París, UNESCO, 1984, p. 15.

[2] J. V. Peris, Conervación y Restauración de material cultural en Archivos y Bibliotecas, Valencia-España: Pentagraf Impresores, S.L., 2002, p. 29.

[3] G. H. y. A. A. Hopkins, «Tintas, Papeles, Lápices, Lacres etc.,» de El recetario industrial, Barcelona, Gustavo Gili, SL, 1994, p. 1179.

[4] O. C. y. M. Lazzari, «Photo-oxidative stability of paraloid acrilic protective polymers,» ELSEVIER, pp. 17-21, 2000.

[5] A. C. Rodríguez, «Barnices sintéticos : estudio comparativo de barnices sintéticos utilizados en la restauración de ceramicas,» vol. 6, n 29-46, 2002.

[6] «UNESCO,»19 Noviembre 2004. [En línea]. Available: http://www.unesco.org/culture/natlaws/media/pdf/ecuador/ec_codificacion_27_ ley_de_patrimonio_cultural_spaorof.pdf. [Último acceso: 7 Julio 2015].

[7] A. E. Q. Valle, «Utilización de residuos de cáscara de naranja para la preparación de un desengrasante doméstico e industrial,» Quito, 2009.

[8] F. Kiekeben, «nontoxicprint,» 2015. [En línea]. Available: http://www.nontoxicprint.com/safesolvents.htm. [Último acceso: 3 Julio 2015].

[9] V. y. C. P. Bernal, «Extracción del aceite esencial de la cáscara de naranja: caracterización y estudio de potencial industria en el Ecuador,» Quito, 2012.

[10] P. M. \&. S. E. Moys, «Aspectos gerais do poliestireno,» vol. 16, 2002.

[11] R. \&. R. H. Navarro, «Plastificantes de todo tipo.» vol. 103(664), nº 14-19, 2012.

[12] S. P. Prades, «Universidad Politeécnica de Valencia,» 15 Enero 2009. [En línea]. Available: https://www.upo.es/moleqla/export/sites/moleqla/documentos/Numero18/Peirx_Pradesx_Sara_-_Molequla_xPilarx.pdf. 